\title{
Paradoxical rapid normalization of serum proteins in severely sarcopenic pediatric patients following liver transplant
}

\author{
Christian M Schmidt II ${ }^{1}$, Richard S. Mangus ${ }^{2}$ \\ ${ }^{1}$ Indiana University School of Medicine, ${ }^{2}$ Indiana University School of Medicine, \\ Department of Surgery, Transplantation Section
}

\section{Background and Hypothesis:}

Pediatric liver disease patients develop frailty which risks adverse outcomes. This novel study aims to quantify differential rates of sarcopenia in pediatric liver failure pre/post-transplant as a function of nutritional parameters and dynamic changes in muscle mass.

\section{Project Methods:}

A retrospective review of a prospectively collected transplant database (20012018) at a single center was performed. Weekly serum protein values (albumin and total protein) and monthly body weights (BWs) and BMls were collected. A select group with CT imaging pre/post-transplant underwent scaled scoring (cross sectional area at L2/L3 intervertebral space divided by height ${ }^{2}$ for core muscle mass). The post-transplant rates of change were compared to pretransplant baseline nutrition status.

\section{Results:}

There were 114 subjects, 82 underwent CT analysis. Pre-transplant, $45 \%$ had severe sarcopenia. Post-transplant, median serum protein levels normalized, but slowest in patients infants. BMI and BW were unchanged except in adolescents it significantly decreased in the first 30 days. Paradoxically, median serum protein levels normalized at week 3 for patients with severe sarcopenia, faster than $\mathrm{mild} /$ moderate sarcopenia groups.

\section{Conclusion and Potential Impact:}

Liver transplant normalizes serum proteins at differential rates according to age, pre-transplant BMI and level of sarcopenia. Despite normalization liver transplant does not change BW and BMI. Paradoxically, severe sarcopenia patients normalize their serum protein levels at the fastest rate. We speculate an increased level of a novel "protein-stimulating factor" may exist in the severely sarcopenic group. Understanding post-liver transplant outcomes in patients as a function of their pre-transplant sarcopenic index is a novel method to optimize these patients care. 